\title{
Globalización y reforma educativa en América Latina: un análisis inter-textual
}

\author{
JORGE M. GOROSTIAGA \\ Universidad Nacional de San Martín \\ CÉSAR G. TELLO \\ Universidad Nacional de La Plata \\ Tres de Febrero y San Martín, Universidad Nacional de Tres de Febrero \\ Universidad Nacional de San Martín
}

\section{INTRODUCCIÓN}

Este artículo aborda la relación entre los procesos de globalización y las reformas educativas que han tenido lugar en América Latina durante las últimas dos décadas. Damos cuenta de una investigación que buscó caracterizar y comparar las principales perspectivas sobre esta temática a través del análisis de textos producidos por académicos y por organizaciones gubernamentales y no gubernamentales. Siguiendo la metodología de la cartografía social, se combinan herramientas del análisis textual con una representación visual del discurso como campo intertextual.

En el mapeo que presentamos, hemos identificado siete perspectivas principales que surgen del análisis de los textos: Economicista, Inserción Imperativa, Integracionista, Humanista, Crítica Normativa, Crítica Analítica y Mundialización Alternativa. Las dos primeras sostienen una visión positiva de la globalización, asociada a una valorización del papel de la educación en contribuir a mejorar la competitividad económica de los países; la tercera es más ambigua, pero también apoya la idea de la adaptación de la educación a los requerimientos globales; las 
restantes destacan distintos aspectos negativos de los procesos de globalización que se han desarrollado en la región y de sus implicancias en términos de reforma educativa. La última perspectiva, además, argumenta a favor de una globalización antihegemónica que pueda ponerse al servicio de la democratización y la justicia social.

\section{METODOLOGÍA}

La cartografía social es una metodología que busca mejorar nuestra comprensión de las cambiantes formas en que se estructuran campos de conocimiento o de discusión de políticas y prácticas sociales (véase Gorostiaga, 2009; Tello y Gorostiaga, 2009). Dada la creciente diversidad de comunidades discursivas que compiten entre sí formulando argumentos y contra-argumentos, este enfoque resulta sumamente apropiado para brindar una interpretación provisoria de las múltiples visiones existentes sobre la educación y la sociedad (Paulston, 1995).

A través del mapeo de un campo discursivo, se busca presentar las cosmovisiones, argumentos y características retóricas de distintas perspectivas que en un análisis más general son pasados por alto o permanecen en una zona oscura, y que implican distintas opciones de política. Esto no supone ignorar que toda política es moldeada también por otros debates y discursos relacionados, así como por las relaciones de poder existentes que resultan en particulares usos o no-usos de argumentos de política.

El análisis de textos y de sus interrelaciones constituye la principal herramienta para la identificación de perspectivas o formas de ver un determinado fenómeno, por lo cual el espacio discursivo asume la forma de un campo intertextual en el que la posición de cada texto se define en relación a los otros textos. Este es un análisis interpretativo en el que "determinados aspectos del texto son puestos de relieve a expensas de otros" (Paulston, 2001, p. 19). Para la identificación y caracterización de las perspectivas, se realiza un proceso iterativo de:

1 | lectura cuidadosa y en profundidad de los textos, con especial atención a los argumentos o ideas centrales;

2 | identificación provisional, a través de la lectura de los textos, de las principales perspectivas;

3 | selección de los textos ilustrativos de cada perspectiva, lo cual puede implicar reducir el número de textos bajo análisis, eliminando aquellos que presentan argumentos ya desarrollados por otros textos;

4 lectura en profundidad de los textos seleccionados enfocada en argumentos, cosmovisiones y características retóricas;

5 | revisión de las perspectivas identificadas;

6 incorporación/eliminación de textos ilustrativos. Esto es, de nuevo, un ejercicio altamente interpretativo y "marcar los límites de las orientaciones 
textuales o las categorías genéricas es, por supuesto, controversial" (Paulston, 1995, p. 174).

Dentro de ese campo intertextual, las perspectivas son mapeadas topográficamente con la ayuda de imágenes o representaciones visuales para proveer un "ordenamiento" siempre provisorio. La representación visual del campo intertextual se realiza a través de un mapa construido en base a las dos dimensiones que surgen como las más significativas en el posicionamiento y diferenciación de las perspectivas identificadas.

En este estudio se analizaron 45 textos (véase la lista completa en el Anexo) publicados entre 1996 y 2008 que discuten las relaciones entre los procesos de globalización y las reformas educativas implementadas en la región durante los últimos veinte años. Para la búsqueda de textos se utilizaron diferentes fuentes bibliográficas (Bases Scielo, EBSCO, REDALYC, Google Académico, Dialnet, Bibliotecas virtuales de OEI y RINACE) y se consultaron bases de documentos de los sitios de organismos internacionales. Se incluyen textos producidos por académicos, en forma de artículos en revistas especializadas, libros, capítulos de libros y conferencias; y documentos elaborados por organizaciones gubernamentales y no gubernamentales de carácter regional, a los que se agrega un texto de un grupo de sindicatos docentes. Nos concentramos en textos que se refieren a América Latina como región o a un conjunto de países, en lugar de a países particulares de la región. Asimismo, hemos dejado de lado textos enfocados exclusivamente en educación superior. El análisis de cada texto se realizó identificando los argumentos sobre 1) qué es la globalización, 2) cuáles son sus efectos generales, 3) el sentido e implicancias de las reformas educativas que se desarrollan en los países de la región, y 4) cómo se interrelacionan ambos procesos (globalización y reforma educativa).

\section{GLOBALIZACIÓN Y EDUCACIÓN}

La globalización puede ser definida como "la intensificación de las relaciones sociales a nivel mundial que vinculan lugares distantes de tal manera que los acontecimientos locales están moldeados por acontecimientos que ocurren a muchos kilómetros de distancia y viceversa" (Held, 1991, p. 9 apud Torres, 2001). Si bien los procesos de interconexión a nivel mundial llevan varios siglos de desarrollo, la globalización contemporánea (post 1945) no tiene precedentes históricos en términos de su extensión, intensidad, velocidad e impacto (Held et al., 1999). Suele aceptarse que "la globalización adopta distintas formas y opera sobre, y a través de, distintas esferas de la vida social: política, económica, cultural y tecnológica” (Robertson et al., 2007, p. 9).

Dentro de las ciencias sociales se distinguen tres enfoques en la discusión reciente sobre los procesos de globalización (Held et al., 1999; Tikly, 2001): el 
"hiperglobalista", según el cual el triunfante capitalismo global hace que los Estados pierdan relevancia con la aparición de nuevos tipos de cultura, gobierno y sociedad civil globales; paralelamente, las formas tradicionales de escolarización están en vías de desaparecer frente a los avances tecnológicos. En segundo lugar, una visión "escéptica", que sostiene que los Estados siguen siendo los que procesan las crisis económicas, en un contexto en el que se agudizan las diferencias entre países centrales y periféricos; en esta visión los sistemas educativos nacionales siguen teniendo preeminencia para explicar los procesos de política educativa, a pesar de una mayor convergencia y de una internacionalización parcial (como la evidenciada en los procesos de movilidad estudiantil).

Por último, un enfoque "transformacionalista" de la globalización (que es el que este artículo adopta) acepta que vivimos una etapa caracterizada por niveles de interconexión global nunca antes experimentados en lo político, lo económico y lo cultural, pero también por procesos contradictorios y complejos que apuntan a fenómenos de fragmentación y estratificación, en los que determinados grupos y regiones sufren una creciente marginalización y en que los conceptos de "centro" y "periferia" cobran nuevas implicancias (Held et al., 1999; Tikly, 2001). Al mismo tiempo, se considera que los estados nacionales, sin abdicar completamente su poder y articulando diferentes respuestas frente a la nueva configuración del sistema mundial, se ven crecientemente forzados a aceptar instancias internacionales que los limitan.

Las reformas educativas a niveles nacionales y sub-nacionales son a la vez una respuesta y un medio a través del cual la globalización impacta en lo social. En los últimos años, tanto los países centrales como los periféricos han tendido a aceptar una "nueva ortodoxia" de políticas educativas (Ball, 1998) que apunta a reforzar las conexiones entre educación, empleo y el mejoramiento de la economía nacional, reducir los presupuestos educativos, establecer controles centrales más directos sobre el curriculum y la evaluación, y buscar mecanismos de elección y descentralización hacia las escuelas. El escenario de política educativa que comienza a definirse en la década de 1980 se caracteriza por la búsqueda de la eficiencia y la calidad, orientado a la formación de recursos humanos que permitan aumentar la competitividad internacional de las economías nacionales (Ball, 1998; Taylor et al., 1997). En las últimas décadas, los discursos y los lineamientos en diferentes lugares del mundo parecen haber tenido un conjunto de ejes comunes: la descentralización, el énfasis en incrementar los niveles de autonomía escolar, la "profesionalización" docente y los mecanismos de evaluación del rendimiento académico (Whitty; Power; Halpin, 1998).

Hacia fines de la década de 1980 y principios de los ‘90, se extendió en America Latina la preocupación por la calidad de la educación junto con la necesidad de impulsar la formación de capital humano para el desarrollo económico y social (Braslavsky, 1999; CEPAL, 1992). La reforma - en muchos casos a través de la sanción e implementación de nuevas leyes generales de educación - fue un 
fenómeno común a casi todos los países latinoamericanos, en un movimiento que proponía los cambios educativos más profundos desde la instauración de los sistemas educativos nacionales de fines del século XIX y principios del XX. Estas reformas se produjeron en el marco de procesos de reestructuración neoliberal del Estado y la economía que incluían la apertura al comercio internacional y el fomento de la inversión extranjera, con el apoyo de diferentes organismos internacionales.

Las principales políticas educativas instrumentadas en la década de 1990 consistieron en la ampliación de los años de escolaridad obligatoria, la profundización de la descentralización administrativa, la promoción de una mayor autonomía escolar, el establecimiento de sistemas de medición de la calidad (rendimiento académico de los alumnos) y de sistemas de información, la modernización curricular, el desarrollo docente y la focalización en los grupos sociales más postergados (Gajardo, 1999; Fischman et al., 2003). Si bien se ha registrado un aumento en la cobertura de los sistemas educativos, no han mejorado los resultados de aprendizaje y persisten, o se han agravado en algunos casos, las desigualdades sociales y regionales (Tenti Fanfani, 2007; Poggi, 2010).

Desde 2002, con cambios en las orientaciones políticas de algunos de los gobiernos de la región, algunas políticas parecen reorientarse hacia una regulación más directa por parte de los estados nacionales y una recuperación de la educación como derecho social. Pulido Chaves (2010) distingue entre tres tipos de políticas educativas a nivel nacional que se estarían dando actualmente en la región: 1) las continuidades neoliberales (México, Perú, Chile desde 2010, Costa Rica, Colombia, entre otros); 2) las rupturas de concertación (Argentina, Brasil, Chile 1990-2009, Guatemala, Uruguay y Paraguay); y 3) las rupturas de autonomía (Bolivia, Ecuador y Venezuela). Según este autor, lo que los modelos de ruptura están marcando, en mayor o menor medida de acuerdo con los diferentes contextos sociales y políticos nacionales, es un avance hacia una concepción democrática y radical del derecho a la educación, en oposición a la visión mercantilista que predominó en los noventa y que se perpetúa en las políticas del primer tipo.

\section{PERSPECTIVAS EN EL DISCURSO SOBRE GLOBALIZACIÓN Y REFORMA EDUCATIVA EN AMÉRICA LATINA}

La Figura 1 presenta un mapa del discurso sobre globalización y reforma educativa en América Latina con las posiciones de las perspectivas identificadas y sus inter-relaciones. El mapa ha sido construido en base a dos dimensiones. La dimensión horizontal se relaciona con la visión de los procesos de globalización, con el enfoque hiperglobalista en un polo y el enfoque escéptico en el otro; de acuerdo con la tipología presentada en Tikly (2001), el centro de esta dimensión estaría asociado al enfoque transformacionalista. La dimensión vertical, por su parte, alude a valorizaciones sobre las reformas educativas y sus fines, con la competitividad económica en un polo y la democratización social en el polo opuesto. Las flechas de 
líneas continuas indican los casos de perspectivas que se apoyan selectivamente en argumentos de otras perspectivas, mientras que las flechas de líneas punteadas indican críticas desde una perspectiva hacia los argumentos de otra. Se identifican también textos que combinan elementos de dos perspectivas, situados por lo tanto en un espacio de intersección.

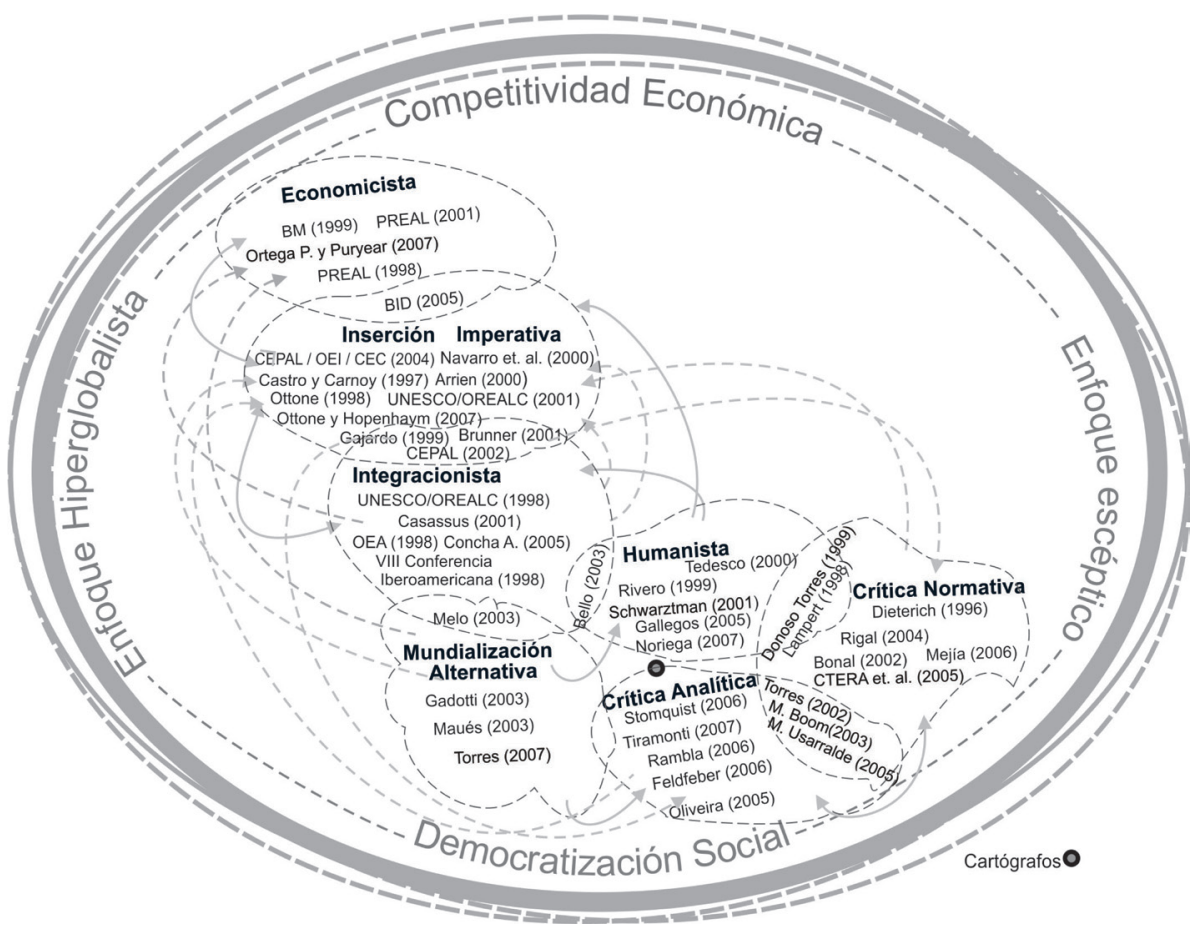

Figura 1.

Un mapeo del discurso sobre las relaciones entre globalización y reforma educativa en América Latina como campo intertextual.

A continuación presentamos una breve caracterización de cada perspectiva con algunos ejemplos de textos ilustrativos.

\section{Economicista}

Para esta perspectiva, la preocupación dominante es la de mejorar la competitividad económica de los países de la región, sin cuestionar el escenario global, y promoviendo un enfoque de reforma educativa en línea con la "nueva ortodoxia" de políticas educativas (Ball, 1998) que hemos discutido en la sección anterior. A través de un tono altamente prescriptivo, se argumenta que las nuevas condiciones económicas "exigen" la adopción de ciertas medidas educativas que posibiliten la plena inserción de los países latinoamericanos en el orden mundial. 
Como uno de los ejemplos de esta perspectiva, tomamos un documento del Banco Mundial (1999) que se enfoca en America Latina y el Caribe. Este texto propone tres metas para la educación: "proporcionar una fuerza laboral capacitada y flexible para bien del crecimiento económico, fomentar la cohesión social y promover la democracia, y reducir las desigualdades sociales y la pobreza" (p. 26). El énfasis está puesto en el desarrollo del capital humano, explicando que:

Una de las principales debilidades de los países de ALC en el mercado mundial actual es la falta de capital humano, lo cual ha limitado severamente su capacidad de dedicarse a la investigación y de introducir innovaciones tecnológicas. Por lo tanto, sus posibilidades de responder al desafío de la competencia internacional dependerán de la rapidez con que puedan diversificar sus economías, actualizar las habilidades de su fuerza laboral actual, y preparar a los niños para adaptarse a las circunstancias económicas cambiantes cuando ingresen al mundo laboral. (p. 28)

El documento resalta "la enorme y creciente brecha" (p. 50) de rendimiento educativo entre la región y los países de la Organización para la Cooperación y el Desarrollo Económico (OCDE).

En lo que podría verse como una respuesta a lo que veremos son, en nuestro mapeo, planteos de las perspectivas que cuestionan las implicancias del orden económico vigente, el Banco Mundial (1999) reconoce que como consecuencia de la globalización y la liberalización han aumentado las desigualdades entre países y entre grupos sociales al interior de las naciones, pero sostiene que estas desigualdades -“consecuencias no deseadas de la globalización”- se resuelven con programas de educación, nutrición y salud para los sectores más pobres.

Encontramos otro ejemplo de esta perspectiva en un documento del Programa de Promoción de la Reforma Educativa en América Latina (PREAL, 2001), el cual enfatiza, en algunos pasajes con un tono dramático, la obligación de "avanzar al ritmo de las necesidades de la economía global” (p. 7-8). A pesar de que se menciona repetidas veces la importancia de la educación para el fortalecimiento de la democracia y el aumento de la equidad, el acento parece puesto en el desarrollo económico y en la competencia con otros países. Se señala que Latinoamérica no está preparando a sus alumnos para competir en una economía globalizada (p. 24). Además, se plantea la necesidad de otorgar poder de decisión a las escuelas, con metas de rendimiento y mecanismos de rendición de cuentas establecidos a nivel central, instando a que éstas participen en pruebas estandarizadas internacionales, ya que "la competitividad internacional exige que cada país tenga una clara comprensión del rendimiento de los alumnos" (2001, p. 3; véase también PREAL, 1998).

Ortega Goodspeed y Puryear (2007), por su parte, argumentan que las escuelas en América Latina no contribuyen a hacer más competitivas las economías 
de la región. Esto se debe a déficits de calidad, cantidad y equidad, particularmente en la educación secundaria y en la superior, los cuales son ilustrados con resultados de pruebas internacionales y nacionales de rendimiento académico, tasas de graduación, etc. Se identifican en este texto cinco factores como causas del bajo desempeño de las escuelas: 1) falta de estándares de aprendizaje y de sistemas sólidos de medición de resultados; 2) enseñanza a cargo de docentes con débil formación, malas condiciones de trabajo y falta de incentivos; 3 ) ausencia de mecanismos de rendición de cuentas en un contexto de débiles conexiones entre escuelas, comunidad local y empleadores; 4) inversión por debajo del promedio de los países desarrollados, en particular en los niveles primario y secundario; 5) la mayor influencia de grupos de interés (como sindicatos docentes y estudiantes universitarios) respecto a los padres, docentes y empleadores en la formulación de las políticas educativas.

\section{Inserción Imperativa}

En esta visión, si bien el argumento económico mantiene un peso considerable, además se aduce que las políticas educativas en la región deben contribuir a la conformación y preservación de una identidad cultural específica y propia. A pesar de que se reconocen tensiones y aspectos negativos de la globalización, se sostiene que los países latinoamericanos deben insertarse en la economía global. La educación, según esta perspectiva, juega un rol clave en la formación de los individuos como trabajadores y como ciudadanos que contribuyan al crecimiento económico y al fortalecimiento de las instituciones democráticas.

El documento de CEPAL/OEI/CE (2004), por ejemplo, presenta un enfoque que intenta diferenciarse de una aproximación puramente economicista y explica que "la educación no sólo forma capital humano; es también una vía de realización humana y un mecanismo de transmisión de los valores sociales que permiten la convivencia" (p. 22). En este texto se plantean temas como el de la gobernabilidad del sistema educativo, la generación de una "nueva ciudadanía", la necesidad de una educación intercultural e inclusiva, y la democratización de la escuela, acompañados por una crítica a las "propuestas a favor de la privatización por parte de organismos multilaterales de crédito" (p. 19). Sin embargo, el objetivo de la "competitividad" aparece como muy central, especialmente expresado en el objetivo de alcanzar "la utopía de una sociedad competitiva y solidaria” (p. 37). A partir de un diagnóstico que se centra en la desigualdad o inequidad (se usan ambos términos) educativa y tecnológica, se retoman conceptos del documento de la CEPAL/UNESCO Educación y conocimiento: eje de la transformación productiva con equidad (1992), y similares a los planteados en CEPAL (2002), postulando a la educación como un factor fundamental para alcanzar crecimiento económico con equidad.

A su vez, el texto argumenta que, en el actual contexto, existen dos agendas de transformación para los países de América Latina: por un lado, es necesario satisfacer "asignaturas pendientes del siglo XX", asociadas a la universalización de 
la cobertura de la educación básica, y el mejoramiento de la calidad y la modernización de todos los niveles, junto con la formación para la ciudadanía. Por otra parte, se requiere adaptar los sistemas educativos a las transformaciones tecnológicas y culturales propias del siglo XXI, sin descuidar el cultivo de la identidad cultural de los pueblos de la región. Para superar el problema de la gobernabilidad del sistema educativo se requiere mejorar la gestión; evaluar resultados de aprendizaje; descentralización territorial; democracia escolar y continuidad de las políticas (CEPAL/ OEI/CE, 2004).

Por su parte, Ottone y Hopenhayn (2007) argumentan que se debe responder desde la educación a la dinámica de la globalización y la sociedad del conocimiento y que esto implica cuatro retos: 1) igualar oportunidades educativas, 2) transformar los procesos de aprendizaje al interior de las instituciones educativas a la luz de las nuevas formas de aprender, conocer, informarse y comunicarse que difunde, a paso acelerado, la sociedad del conocimiento, 3) aumentar la pertinencia de las destrezas que se transmiten en la educación para hacer frente al nuevo tipo de sociedad, refiriéndose a la sociedad del conocimiento y 4) responder a la urgencia de las economías nacionales por insertarse en el concierto global de manera tal que genere círculos virtuosos entre diversificación, crecimiento económico, generación de empleo y efecto pro equidad del crecimiento.

Los autores explican que el insertarse en la dinámica global es una deuda pendiente que las reformas educativas en América Latina no han logrado resolver. En este sentido, plantean que el sistema educativo debe contribuir a la competitividad de la producción interna, tanto de bienes como de servicios. Elevar la productividad de manera masiva, y a la vez generar grandes saltos en aquellos espacios con ventajas comparativas en la economía global, son tareas que la educación no puede perder de vista en su rol de formación de las nuevas generaciones.

Los textos de Arrien (2000), Castro y Carnoy (1997), Navarro et al. (2000), Ottone (1998) y UNESCO/OREALC (2001), con diversos énfasis y cubriendo diferentes aspectos de las reformas educativas en la región, son también ilustrativos de esta perspectiva.

Como ejemplo de la combinación de elementos de las perspectivas Economicista y de la Inserción Imperativa, un texto del Banco Interamericano de Desarrollo (BID) (2005) expresa que la preocupación por reformar y mejorar los sistemas educativos de la región está basada en "cerrar la brecha entre ellos (los países de América Latina y el Caribe) y sus competidores" (p.21), en el marco de lo que se considera un contexto de aumento de la competencia internacional y de las necesidades de habilidades centradas en el conocimiento para el desarrollo de las economías nacionales: "Las exigencias de la economía del conocimiento requieren inversiones a todos los niveles y en todo tipo de educación y de capacitación” (p. 22, énfasis nuestro), además de regulaciones e incentivos que aseguren la calidad de la oferta educativa, y de la atención a aspectos curriculares descuidados como la enseñanza en ciencias y matemáticas. 


\section{Integracionista}

Esta perspectiva manifiesta una visión de la globalización cercana a la concepción transformacionalista y un tono menos aseverativo, en contraposición al optimismo prescriptivo de las perspectivas Economicista y de la Inserción Imperativa. Se pone el acento en la conformación de un espacio regional guiado por un conjunto de ideas comunes que se traducen en similares medidas de reforma educativa en los distintos países. Sin embargo, sigue presente la idea de la adaptación de los sistemas educativos de la región a las tendencias globales, particularmente a través del uso de las nuevas tecnologías de la información y de innovaciones en la organización y gobierno de la educación. Al mismo tiempo, se remarca la necesidad de regulaciones que contrarresten los efectos negativos de la globalización, y de políticas educativas que no se centren sólo en aumentar la eficiencia y el rendimiento académico de los alumnos.

El documento de la VIII Conferencia Iberoamericana de Educación (1998) reconoce a la globalización como "un proceso amplio, contradictorio, heterogéneo" que presenta tanto oportunidades como riesgos. Se sostiene que "es necesario responder a los requerimientos de la globalización por medio de la promoción de la educación a lo largo de la vida y la profundización de la intercomprensión, con vistas a una ciudadanía participativa y responsable”, pero no se explicita cuáles son esos requerimientos ni cuál es la relación entre globalización y sociedad del conocimiento (planteado como otro de los conceptos centrales del documento). Asimismo, se argumenta a favor de la cooperación y la integración entre los países iberoamericanos como una estrategia para hacer frente a los desafíos de la globalización. Las propuestas para los sistemas educativos apuntan a la formación de "ciudadanos responsables, solidarios, competentes y con capacidad suficiente de adaptación a los cambios" y, entre otras, consisten en favorecer la movilidad de las personas, atender a la multiculturalidad, introducir las nuevas tecnologías de la información y la comunicación, promover la equidad y establecer sistemas de medición de la calidad.

Casassus (2001) parte de la dimensión de la globalización que enfatiza la desterritorialidad y la emergencia de espacios supra-nacionales que impactan sobre la esfera cultural, y reconoce que aunque puede concebirse como un fenómeno con más de 500 años, lo que es nuevo es la ampliación de áreas afectadas y la velocidad con que se desarrolla la globalización. En la evaluación de diez años de reforma educativa en la región, el texto destaca la centralidad de la educación como parte de las estrategias de desarrollo; la implementación generalizada de un nuevo modelo de gestión que combina la descentralización de cuestiones operativas con la centralización de las decisiones de política, la evaluación y el curriculum; y acciones para mejorar la calidad, como el desarrollo de sistemas de evaluación, programas de 
"discriminación positiva" y aumento de la autonomía escolar, aunque reconoce que es difícil saber si estas acciones han cumplido con los efectos que se proponían. Más aun, se señala que el discurso de la reforma se ha concentrado en la gestión, relegando la cuestión de los fines de la educación, cuya redefinición "se torna urgente" en la medida en que la educación es percibida como un "instrumento de acceso a la modernidad y a la globalización" (p. 27).

Otros textos que reflejan esta perspectiva son los de UNESCO/OREALC (1998), Concha Albornoz (2005), OEA (1998) y Melo (2008). UNESCO/ OREALC (1998) rescata la idea de una ética global basada en la cooperación entre los pueblos y enfatiza la importancia de los procesos de integración regional y sub-regional como una respuesta a los desafíos de la globalización. Señala que la UNESCO ha promovido en la región "la transformación de la educación superior, para convertirse en factor pro activo en el contexto y proceso de globalización” (1998, p. 53). En relación a los procesos de reforma educativa de la década de los 90, el panorama no es completamente positivo:

Los países han logrado consensos en torno a las acciones necesarias para conseguir una educación de mayor calidad, sin embargo, es preciso avanzar hacia una mayor relación entre calidad y equidad, fortaleciendo la Educación Pública y el rol estratégico del Estado para asegurar la igualdad de oportunidades. En este sentido, es fundamental que en los procesos de descentralización se dote a las escuelas de las condiciones y recursos necesarios para realizar su tarea educativa con la estrecha colaboración de la comunidad... [Las reformas] no llegan en muchos casos a modificar los procesos de enseñanza-aprendizaje que tienen lugar en la sala de clase. (p. 36)

Melo (2008), en tanto, realiza una evaluación positiva de las estrategias regionalistas para abordar los desafíos de una globalización "incierta, turbulenta, compleja” y, particularmente, acerca de cómo el tema educativo ha sido considerado en el trabajo de las Cumbres de las Américas. Sin embargo, este texto también sostiene que los académicos "debemos colocarnos al lado de una globalización alternativa y no de globalización homogeneizadora” (p. 118), lo que lo acerca a la Perspectiva de la Mundialización Alternativa (ver abajo).

Combinando elementos de las perspectivas de la Inserción Imperativa e Integracionista, situamos a los textos de CEPAL (2002), Gajardo (1999) y Brunner (2001). Este último sostiene que se suele utilizar el concepto de globalización para pretender explicar "prácticamente todo" lo que sucede en la educación, sin incluir demostraciones de estas relaciones causales (p. 9). Brunner ataca lo que puede ser visto en nuestro análisis como una de las estrategias argumentativas de la Perspectiva Crítica Normativa (desarrollada más abajo): 
Más bien, puede alegarse que esa alta propensión de impacto atribuida a la globalización en el ámbito educacional se debe, ante todo [...] a la "sobredeterminación ideológica de la globalización”, que consiste en adjudicarle a ésta una orientación ideológica unívoca (neoliberal) y a ésta, a su vez, un efecto en la educación y las políticas educacionales. (p. 11)

A la vez que critica "la tesis de los grandes efectos" que implica atribuirle a la globalización este tipo de influencia, el texto se inclina por una definición de globalización ceñida a "los procesos que resultan de la difusión de las NTIC [Nuevas Tecnologías de la Información y la Comunicación], particularmente las tecnologías de redes" (p. 13). Se señala que estos procesos tienen un impacto significativo sobre los contextos inmediatos de la educación, tales como el acceso a la información, manejo de conocimientos, relación con el mercado laboral, empleo de tecnologías y socialización en la cultura de la época. En suma,

[...] la educación latinoamericana debe dar el salto hacia el siglo XXI y emprender las nuevas tareas de las cuales dependen el crecimiento económico, la equidad social y la integración cultural, adaptando para ello sus estructuras, procesos y resultados y las políticas educacionales a las transformaciones que por efecto de la globalización - experimentan los contextos [...] en que se desenvuelven los procesos de enseñanza y aprendizaje. (p. 32)

Estas tareas incluyen conformar un sistema educativo con estructura de redes e integrar plenamente las NTIC a las aulas, sin descuidar las tareas pendientes de mejorar el acceso a los distintos niveles y el rendimiento en torno a las competencias básicas, con una participación cada vez más activa de la sociedad civil.

\section{Humanista}

A diferencia de las perspectivas anteriormente descriptas, aquí no se plantea una simple adaptación de la educación a las nuevas condiciones sociales y económicas, sino generar cambios que permitan potencializar las capacidades humanas en forma equitativa, denunciando aquellos aspectos de los procesos de globalización que atentan contra esta potenciación. Particularmente se pone el acento en la necesidad de superar - a través de políticas globales ya que la educación por sí sola no alcanza - la desigualdad y las nuevas formas de exclusión social en la región, cuyo agravamiento se considera como un producto de la globalización económica.

El trabajo de Tedesco (2000) argumenta que vivimos una "crisis estructural", que se manifiesta en un "un proceso de profunda transformación social" que implica la aparición de "nuevas formas de organización social, económica y política" (p. 11). En el nuevo escenario social, el conocimiento y la información se convierten en las "variables clave de la generación y distribución del poder en la sociedad" (p. 12). Se señala que el concepto de ciudadanía se ha transformado con el auge de entidades 
supranacionales y el resurgimiento del comunitarismo local (p. 31). A su vez, la globalización económica ha reducido "la capacidad de los estados nacionales para mantener los niveles de beneficios sociales" (p. 32). A los aspectos económicos se suma la globalización de las comunicaciones: "las nuevas tecnologías de la información han provocado el debilitamiento de la capacidad de los estados para controlar el flujo de información" dentro de sus fronteras (p. 35-36).

El texto explicita un rechazo a "los enfoques conservadores" que justifican la desigualdad social, y enfatiza la opción por un nuevo pensamiento democrático que conjugue igualdad y respeto a la diversidad. Se aboga por democratizar el acceso al conocimiento y formar para "una ciudadanía activa”. Deslizándose por momentos hacia un tono normativo, se señala que "el papel de la escuela debe ser definido por su capacidad para preparar para el uso consciente, crítico, activo, de los aparatos que acumulan la información y el conocimiento" (p. 68), y se rescata "la idea de red" que combine autonomía y vinculación de las escuelas.

Otro trabajo que ilustra esta perspectiva es un libro dedicado a repasar con bastante detalle muchas de las iniciativas de reforma educativa de los años noventa en relación a la desigualdad y la exclusión en América Latina, integrando aportes de muy diversas fuentes bibliográficas. En este texto (Rivero, 1999) se considera que "la globalización [...] constituye un proceso disparejo y hasta contradictorio" (p. 8) que se expresa en tres dimensiones complementarias: una económica (concentración del capital, predominio del capital especulativo, industrias del conocimiento), otra cultural y otra geopolítica, aunque "suele ser asumida en Latinoamérica con un enfoque fundamentalista, identificándosele con el liberalismo económico imperante con predominio del mercado y del individuo, dando mayor peso a la apertura comercial, a las privatizaciones y al debilitamiento del Estado en sus distintas funciones". Contra ese "enfoque fundamentalista", en el prólogo del libro se subraya que lo que caracteriza al desarrollo humano no es la elevación del ingreso per cápita, sino el aumento en la cantidad y la calidad de oportunidades para el ser humano.

Rivero afirma que "el discurso de la globalización y el dominio de la lógica del mercado han influido en la vigencia de la retórica del capital humano", retórica auspiciada por las organizaciones multilaterales de crédito y caracterizada por subordinar lo educativo a lo económico y por dejar de lado la idea de la movilidad social (p. 116). Comentando el documento de CEPAL de 1992, y en lo que puede verse como una crítica a la Perspectiva de la Integración Imperativa, Rivero indica que "se señala que los teóricos de la CEPAL aceptan como inevitable el nuevo orden neoliberal y tratan de construir un 'rostro humano' para las relaciones sociales dentro del modelo" (p. 125).

En el final del libro, Rivero critica a los países de la región por no invertir lo suficiente en educación y por no facilitar canales de diálogo y participación ciudadana en los procesos de reforma. Además, pone el acento en la necesidad de no descuidar los aspectos culturales frente al proceso de globalización económica: "La 
estimulación de la heterogeneidad cultural en la educación será primordial para evitar que la universalización del mercado uniformice las expresiones culturales" (p. 285).

Los trabajos de Gallegos (2005), Noriega (2007) y Schwartzman (2001) constituyen otros ejemplos de esta manera de ver la relación entre globalización y reformas educativas en la región. En tanto, un libro de Bello (2003) comparte elementos de las perspectivas Humanista y de la Integración Imperativa. Pone énfasis en los recursos humanos como constitutivos cada vez más de la ventaja comparativa entre los países (p. 48) y subraya que "los planteamientos coinciden en que una de las fórmulas que pueden conducir con éxito a los países latinoamericanos (entre otros que comparten sus características sociopolíticas y socioeconómicas) radica en una adecuada reforma educacional, la cual debe cumplir algunos objetivos básicos enmarcados dentro de las exigencias impuestas por la globalización" (p. 49-50). Bello hace referencia a la importancia de la educación para el fortalecimiento de la democracia: "Para nosotros, la ecuación entre desarrollo y democracia ha constituido un largo y tortuoso camino" (p. 26). Se apela a "una verdadera democratización de Naciones Unidas" para poner bajo su autoridad "a aquellas organizaciones, nacidas de su seno, como el Banco Mundial y el Fondo Monetario Internacional” dado que, plantea la autora, se mantienen muy alejadas de su mandato de estar al servicio de los pueblos, de su desarrollo integral, y no al del mero beneficio del capital y las finanzas internacionales (p. 49-50).

\section{Crítica Normativa}

Considera a la globalización como la expresión de los intereses de los grupos dominantes tanto a nivel internacional como de cada país. Postula que, desde los años noventa (y aun antes), la reforma educativa en América Latina ha sido guiada por la ideología neoliberal, la cual es funcional a dichos intereses, y promovida por las organizaciones multilaterales. Se parte de una visión de conflicto entre distintos grupos sociales con diferentes intereses, tanto a nivel global como a nivel de sociedades nacionales, donde la "lucha" y la "resistencia" de los grupos subalternos - donde se ubica a los docentes y sus organizaciones sindicales - resultan fundamentales para la transformación social.

Desde esta perspectiva, Dieterich (1996) afirma que la globalización expresa en nuestro tiempo "la necesidad expansionista de la sociedad burguesa", manifestada primero en el colonialismo y luego en el imperialismo (p. 60). En un ataque a la aplicación en América Latina de las concepciones de "capital humano", propias de las perspectivas Economicista y de la Integración Imperativa antes discutidas, se señala que "Es ciertamente demagógico sostener que la miseria latinoamericana sea el resultado de la deficiente educación del subcontinente, cuando hay una serie de variables determinantes de igual o mayor importancia”, como la deuda externa, la corrupción de las elites, el proteccionismo del primer mundo, la pobreza y extrema 
desigualdad etc. (p. 80). Uno de los principales argumentos es que desde la década de 1980 la reforma educativa en la región ha estado orientada a "crear al trabajador adaptable que [...] encaje en cualquier 'enchufe' empresarial regional, sin causar problemas en la generación de ganancias" (p. 132).

En el mismo sentido, el texto de Bonal (2002) explica que la agenda del Banco Mundial (BM) y la extensión del neoliberalismo en América Latina han producido un crecimiento de la deuda externa y han dejado a muchos países sin otra alternativa que la de aceptar las condiciones impuestas por el FMI, el BID y el BM para acceder a nuevos créditos que, en gran medida, servirán para financiar la deuda. El programa del BM - dice este autor - ha fracasado, pues generó más pobreza, exclusión y endurecimiento de la calidad de vida, aunque el BM acuse a los propios gobiernos de los países menos desarrollados del fracaso. Afirma Bonal que el interés de este organismo multilateral por el mantenimiento del paradigma neoliberal no es exclusivamente resultado del convencimiento científico e ideológico de la institución, sino que tiene que ver con el hecho de seguir ofreciendo los mejores tipos de intereses en el mercado internacional y con cierto temor a perder su posición hegemónica para imponer un modelo de desarrollo que tiene la influencia directa de los Estados Unidos pese a la imagen de "imparcialidad política" que pretende mostrar el BM.

En la misma línea, un documento en el que sindicatos docentes de Argentina, Brasil, Chile y Uruguay (CTERA et al., 2005) realizan un análisis de las reformas educativas conducidas en sus países durante la década de 1990 denuncia que los sistemas de educación de Latinoamérica han sufrido grandes transformaciones, con frecuencia bajo la presión de instituciones internacionales como el FMI, el BID y el $\mathrm{BM}$. La globalización es vista como un proceso hegemonizado por los organismos multilaterales financieros, expresando los intereses de elites internacionales y locales a través de políticas neoliberales y neoconservadoras. Estas políticas, sostiene el texto, incluyen la liberalización económica, la privatización de las empresas estatales y la desregulación laboral. En el plano educativo, esta hegemonía se traduce en la "imposición" de determinadas políticas, por lo general inspiradas en la economía de mercado, "con mínimas adaptaciones a las particularidades nacionales" (p. 25) y que tienden a profundizar las desigualdades existentes:

El contexto donde se desenvolvieron las reformas educativas del Cono Sur fue el proceso de globalización, el cual está caracterizado por la profundización del desarrollo capitalista mundial a través de la transnacionalización del capital. Las consecuencias de este proceso son la concentración y centralización del capital, así como la consolidación y ampliación de las desigualdades estructurales. (p. 26)

Como respuesta a este proceso, se propugna una transformación radical de la sociedad y se resalta el papel protagónico que los movimientos sociales deberían jugar en ella. 
También los textos de Mejía (2006) (aunque éste más cerca de la Mundialización Alternativa) y Rigal (2004) pueden ser considerados ejemplos de esta perspectiva. Donoso Torres (1999) y Lampert (1998), por su parte, son trabajos que comparten elementos de las perspectivas Humanista y Crítica Normativa. El texto de Donoso Torres asocia la globalización al advenimiento de la sociedad post-industrial, en la cual el conocimiento se convierte en un factor clave para la competitividad de las naciones y donde el mercado asume un espacio de importancia mayor de la mano de la expansión de las empresas transnacionales. Entre los efectos de la globalización se cuentan el achicamiento y ajuste del Estado, el desmantelamiento de las redes de protección social, la declinación de los derechos sociales, el aumento de la pobreza y el aumento de la asimetría entre países centrales y periféricos. Como consecuencia, "los excluidos del nuevo pacto de dominación establecido a través del mercado quedan sujetos a una drástica indefensión que los priva de la capacidad de demandar la presencia del Estado en resguardo de legítimos intereses" (p. 64).

Las reformas educativas de la década de 1990 en América Latina son consideradas "muy afines con el proceso globalizador" (p. 97). La homogeneidad en el tipo de políticas implementadas se origina en la influencia que los organismos internacionales, en particular la CEPAL a través de su documento de 1992, han tenido en la región: "Basta con estudiar con detenimiento lo que recomiendan las agencias internacionales para darse cuenta de que las diferencias de la Reforma Educativa, entre país y país, se deben sólo a necesarias adaptaciones a cada realidad $[\ldots]$ las reformas [...]tienen en común los señalamientos de la CEPAL-UNESCO" (p. 98). El texto señala que "la política educativa propuesta por la globalización de la economía y la sociedad, es el tema de la competitividad y su corolario inevitable, la productividad" (p. 181), temas que "lógica y materialmente son incompatibles" con las aspiraciones de paz y democracia (p. 183).

\section{Crítica Analítica}

La globalización es abordada como un fenómeno que enmarca los cambios educativos de las últimas décadas. El énfasis, a diferencia de la Crítica Normativa, no está puesto en los juicios de valor sobre el proceso de globalización y sus consecuencias sino en la explicación de los mecanismos e implicancias de las reformas implementadas, usando un tono menos taxativo. Esta distinción entre ambas perspectivas no pretende ignorar el componente analítico que existe en la Crítica Normativa ni el aspecto normativo que la Crítica Analítica también posee.

Desde esta perspectiva se postula que las reformas educativas en América Latina han seguido tendencias globales que responden a una variedad de factores y que articulan los intereses de diferentes actores. Las políticas educativas han tendido a aceptar los imperativos de la inserción global, las nuevas formas de regu- 
lación y la adopción de nuevas tecnologías, ignorando las necesidades regionales, las demandas de democratización de la educación y cuestiones de justicia social, incluyendo temas de género.

Oliveira (2005) toma ideas de Santos (2002) y de Dale (2001) para caracterizar a la globalización hegemónica como un conjunto de dispositivos político-económicos que aseguran el orden capitalista e imponen "un ritmo más intenso de producción y reproducción de la vida social” (p. 761). La globalización se asocia a nuevas formas de regulación social que impactan en lo educativo y en las políticas del sector en los países de América Latina. En la regulación educativa que prevalece en la región influyen tanto los organismos internacionales, como procesos políticos y movimientos sociales locales, y elementos estructurales propios de cada país. La educación continúa siendo un importante factor de regulación social, promoviendo la cohesión social y gestionando aspectos del trabajo y de la pobreza en la región. En términos de las reformas instrumentadas,

[...] se destaca la centralidad de la administración escolar, designando a la escuela como núcleo de planeamiento y gestión; el financiamiento per capita [...]; la regularidad y ampliación de los exámenes nacionales; la evaluación institucional y de desempeño [...]; y otros mecanismos de gestión escolar que insisten en la participación de la comunidad (sobre todo auxiliando en cuestiones administrativas), así como la proliferación de consejos con funciones consultivas. (p. 768)

Una consecuencia importante de estas reformas es la restructuración del trabajo docente, ampliado a cuestiones de gestión y planificación, pero en ausencia de las condiciones materiales que hagan posible una real profesionalización y autonomía de los maestros en la organización de su trabajo.

Un texto de Tiramonti (1997) sostiene que en las últimas décadas se produjo un cambio que estuvo orientado a modificar el modelo de desarrollo imperante e iniciar un proceso de reestructuración de la economía tendiente a incorporar a la región al flujo del intercambio mundial de bienes y servicios. Dentro de este proceso, los países de la región inician un proceso de reconversión económica para adaptarse como una necesidad imperiosa. De este modo, la modernización apareció como una exigencia insoslayable para evitar quedarse al margen del "movimiento de la historia”, urgencia que poseía un carácter netamente economicista:

Los sistemas educativos de la región se encuentran, a nuestro entender, sobre-determinados por este contexto de globalización, desintegración y reconversión del lugar social del Estado. Sostendremos en este texto que los sistemas están tensionados entre el imperativo de la inserción [al orden económico global], la reconstitución de los dispositivos regulatorios del Estado y la demanda por la integración societal. 
El modelo de reforma implementado establece una articulación positiva entre autonomía, calidad y equidad. "En el campo educativo se trata de pasar de políticas centradas en el principio de la equidad o la igualdad de oportunidades a un conjunto de acciones" que se organizan alrededor de la competitividad y que prescribe para el Estado una acción destinada a introducir una lógica de cálculo racional-instrumental (de medios y fines y costos y beneficios) en la organización del sistema. La autora plantea al final del artículo que las reformas educativas de los '90 instituyeron lógicas y valores de los cuales se generan pautas de acción cotidiana de la escuela y procesos de socialización a través de los cuales se moldean subjetividades propensas a la aceptación del veredicto del mercado.

El texto de Feldfeber (2006), por su parte, define claramente la relación entre el proceso de globalización y las reformas educativas implementadas en América Latina en las últimas décadas:

Las nuevas tendencias en materia de política educativa, orientadas por el objetivo de la formación de recursos humanos competitivos, parten de un diagnóstico que da por sentado el proceso de globalización y la necesidad de insertarse "creativamente" en este proceso sin cuestionar la forma que asume en el contexto actual. (p. 16)

Se adopta una postura cercana a la visión transformacionalista de la globalización, al señalar algunas de sus contradicciones y al postular que, sin tratarse de un proceso novedoso, ha adoptado características específicas en los últimos tiempos. También se muestra cómo los organismos internacionales (desde el BM hasta la CEPAL) promueven el paradigma de la Sociedad del Conocimiento como una supuesta superación de las desigualdades y como el camino para la inserción en los procesos de globalización. Cuestionando directamente los argumentos de las perspectivas Economicista, de la Inserción Imperativa e Integracionista, Feldfeber (2006) sostiene que en la mayoría de los países latinoamericanos, dedicados primordialmente a la producción de bienes de bajo valor agregado, "una mayor capacitación de la gente no impacta directamente en el empleo y en la competitividad de la economía” (p. 19).

Otros trabajos que reflejan esta perspectiva son los de Rambla (2006) y Stromquist (2003), mientras que Torres (2007), Martínez Boom (2003) y Martínez Usarralde (2005) pueden ser vistos como situados en un espacio de intersección entre las dos perspectivas críticas.

\section{Mundialización Alternativa}

Comparte algunos de los argumentos sobre las tendencias de reforma educativa de las perspectivas críticas, pero sostiene que una globalización alternativa - opuesta a la dominante y basada en la justicia social, la democratización y el desarrollo de la educación como derecho social - ya está siendo promovida por organizaciones sociales en América Latina. 
Gadotti (2003), por ejemplo, señala que existen varios procesos de globalización entre los que se destacan dos: la globalización del modo de producción capitalista a través de la economía de mercado, el cual "extendió un modelo de dominación económica, política y cultural totalitaria y excluyente" (p. 98, itálicas en original); y la globalización de la sociedad civil, producto de "los avances tecnológicos que crean las condiciones materiales (no éticas ni políticas) de la ciudadanía global" (idem, ibídem). Postula la idea de una "ciudadanía planetaria", para "destacar la pertenencia al planeta y no al proceso de globalización” (p. 100), el cual está más identificado con el modelo económico político neoliberal. Enfatizando la posibilidad de una "globalización cooperativa y solidaria" (p. 101), ligada a la sustentabilidad ecológica, la democratización y la superación de la desigualdad económica, Gadotti argumenta que:

Frente al fenómeno de la globalización, no podemos comportarnos ni como los apocalípticos que ven en la globalización la fuente de todos los males actuales, ni como los integrados, que ven en esta la salvación o la condición final de la realización plena del ser humano. (p. 99)

Maués (2003), por su parte, toma uno de los aspectos fuertes de la reforma planteando como tesis principal de su texto que la reforma de la formación de profesores es una tendencia internacional, ligada a las exigencias de los organismos internacionales. Esto se da porque la globalización, el neoliberalismo y los organismos internacionales imponen ciertos marcos referenciales para determinar políticas educativas en el mundo; los países se ven obligados a cumplir con estos marcos referenciales para no quedar "fuera de juego". Y estos marcos referenciales poseen objetivos bien claros:

As reformas educacionais, a partir do final da década de 1980, partiram dos mesmos princípios: as mudanças econômicas impostas pela globalização, exigindo maior eficiência e produtividade dos trabalhadores, a fim de que eles se adaptem mais facilmente às exigencias do mercado. (p. 94)

Paralelamente, otro fenómeno propio de estos tiempos es el de la mercantilización de la educación, que también impacta directamente las políticas de formación y profesionalización docente y sobre otras políticas y practicas educativas. La autora concluye que se necesita una salida al modelo neoliberal y plantea otra globalización: sin exclusiones, sin división de países en el desarrollo y sin analfabetos.

El texto de Torres (2007) resalta tanto los nuevos espacios de formación que exceden a la escuela, como la multiplicidad y contradicciones del fenómeno de la globalización, destacando cinco de sus formas actuales: 1) la neoliberal (intercambio comercial y financiero, privatización), 2) la globalización desde abajo (vinculada a la planetarización de la que habla Gadotti, 2003), 3) la internacionalización de 
culturas y sociedades, 4) la globalización de los derechos humanos, y 5) la globalización de la guerra internacional contra el terrorismo (librada principalmente por los Estados Unidos):

La globalización neoliberal ha tenido impacto en tres aspectos de la educación latinoamericana (Torres, 2007):

a nivel de la economía política del financiamiento educativo, promoviendo formas de privatización, financiamiento a la demanda y escuelas autogestionadas (siguiendo en muchos casos el modelo chileno desarrollado en el gobierno de Pinochet);

en términos de las vinculaciones entre educación y trabajo, las cuales se complejizan por las transformaciones de los mercados de trabajo y por las posibilidades de movilidad del capital;

y mediante la creación de un movimiento pro estándares de excelencia académica internacional, "usado más como instrumento de control político que como herramienta de mejoramiento educativo" (p. 12) y que pone el acento en los mecanismos de evaluación, desvirtuando la tarea docente.

Después de reivindicar la ecopedagogía de Gadotti, Torres plantea la necesidad de "una educación para la justicia social" que cuestione las desigualdades, el individualismo y la mercantilización de la educación y que ponga al conocimiento al alcance del público y al servicio de una democracia radical. Basándose, al igual que Gadotti, en el legado freireano, se postula que:

En el contexto de la lucha contra la globalización neoliberal, la respuesta está en promover una planetarización respetuosa y digna, de los hombres y mujeres de este planeta, basada en una ética del trabajo, la comunicación y la solidaridad, pero también una ética de la producción que no esté fundada en la codicia, avaricia o usura. (p. 16)

\section{CONCLUSIONES}

Nuestro mapeo del discurso sobre la relación entre globalización y reforma educativa en América Latina nos ha permitido identificar siete perspectivas o formas de ver el fenómeno en cuestión que revelan diferentes cosmovisiones y argumentos. La Economicista propone la adopción de políticas neoliberales que mejoren la competitividad económica de los países de la región sin cuestionar en absoluto las tendencias globales. Para las perspectivas de la Inserción Imperativa y la Integracionista, la competitividad económica sigue siendo importante, pero, con distintos matices, se reconocen efectos negativos de la globalización y se resaltan aspectos 
políticos y culturales de los procesos educativos. En la Perspectiva Humanista encontramos fuertes críticas a las consecuencias de la globalización, en particular el aumento de la desigualdad y el surgimiento de nuevas formas de exclusión. Las perspectivas Crítica Normativa y Crítica Analítica tienden a concentrar sus ataques en la mercantilización de la educación asociada al proceso de globalización económica, así como el rol de los organismos internacionales en promover reformas que no responden a los intereses de los sectores mayoritarios de la población ni a las realidades propias de la región. La Perspectiva de la Mundialización Alternativa, por último, plantea la posibilidad de una "planetarización" desde los actores de base y las organizaciones sociales y al servicio de la justicia social, opuesta a las tendencias neoliberales de la globalización económica.

Es de destacar que ninguna perspectiva se muestra conforme con los resultados de las reformas educativas implementadas en la región en los últimos años. La respuesta a cuál es la dirección que las políticas y las prácticas deben tomar en el actual contexto global difiere de acuerdo con cada uno de los puntos de vista y - más allá de un aparente consenso en la necesidad de invertir más recursos en el sector educativo - se enfatizan distintas medidas: el establecimiento de mecanismos de medición de resultados y de rendición de cuentas (P. Economicista); el desarrollo y acceso a las nuevas tecnologías de la información (P. de la Inserción Imperativa y P. Integracionista); el respeto de la diversidad socio-cultural y la formación de sujetos críticos (P. Humanista); y la promoción de la democratización educativa (perspectivas críticas y de la Mundialización Alternativa). Desde nuestro propio posicionamiento, el cual está presente en todo el análisis realizado y representado en la Figura 1 en el lugar de los cartógrafos, resultaría auspicioso que el discurso sobre la globalización y las reformas educativas latinoamericanas profundizara en las formas y mecanismos que la democratización puede adoptar en los distintos países, así como en las instancias concretas de mundialización existentes y posibles, teniendo en cuenta en todo momento la diversidad de contextos que la realidad de nuestra región ofrece.

Tal como señalamos anteriormente, desde mediados de la década del 2000 se hace evidente cierta ruptura en las políticas educativas de algunos países de la región, que podrían asociarse al agotamiento del neoliberalismo como modelo de desarrollo y al surgimiento de una globalización post-neoliberal (Santos, 2008). Dado que nuestro análisis incluye textos publicados hasta el año 2008, es posible prever el surgimiento de nuevas perspectivas en el campo intertextual que den cuenta de los cambios, así como de nuevas interpretaciones que propongan otros mapeos que nos ayuden a mejorar nuestra comprensión de la naturaleza e implicancias de las reformas educativas latinoamericanas. 


\section{REFERENCIAS}

BALL, Stephen. Big policies, small world. Comparative Education, v. 34, n. 2, p. 119-30, 1998.

Braslavsky, Cecilia. Re-haciendo escuelas. Buenos Aires: Santillana, 1999.

CEPAL/Unesco. Educación y conocimiento: eje de la transformación productiva con equidad. Santiago de Chile: Oficina Regional de Educación de la UNESCO para América Latina y el Caribe, 1992.

DALE, Roger. Globalização e educação: demonstrando a existência de uma cultura educacional mundial comum ou localizando uma agenda globalmente estruturada para a educação. Educação, Sociedade E Culturas, Porto, n. 16, p. 133-69, 2001.

Fischman, Gustavo; Ball, Stephen; Gvirtz, Silvina. Toward a neoliberal education? Tension and change in Latin America. In: BALL, Stephen; Fischman, Gustavo; GvirTZ, Silvina (Org.) Crisis and Hope: The Educational Hopscotch of Latin America. New York: Routledge Falmer, 2003.

Gorostiaga, Jorge. La cartografía social: aportes al análisis del discurso en educación. In: PINI, Mónica (Org.) Discurso y educación: herramientas para el análisis crítico. Buenos Aires: UNSAM Edita, 2009.

Held, David; Mcgrew, Anthony; Goldblatt, David; Perraton, Jonathan. Global Transformations: politics, economics, culture. Cambridge: Polity, 1999.

HeLD, David. Political theory today. Stanford: Stanford University Press, 1991.

Paulston, Rolland G. El espacio de la educación comparada y el debate sobre el posmodernismo. Propuesta Educativa, Buenos Aires, n. 23, p. 18-31, 2001.

. Mapping knowledge perspectives in studies of educational change. In: Cookson, Peter W. Jr.; Schneider, Barbara (Org.) Transforming Schools. New York: Garland, 1995.

Poggi, Margarita. Una radiografía de los sistemas educativos de América Latina. Desafíos para las políticas educativas. Pensamiento Iberoamericano 7, p. 3-25, 2010.

Pulido chaves, Orlando. Sobre el derecho a la educación en América Latina. Mesa sobre los contextos regionales y el derecho a la educación, Pre-Asamblea Latinoamericana de la Campaña Mundial por el Derecho a la Educación. Bogotá, noviembre 2010. Disponible en: <http://educacionenelmundo.wordpress.com/2010/12/03/analisis-sobre-el-derecho-a-la-educacion-en-america-latina>. Acceso: diciembre 2010.

RoberTson, Susan; Novelli, Mario; DALE, Roger; TikLy, Leon. Globalisation, Education and Development: ideas, actors and dynamics. Londres: Department for International Development, 2007.

Santos, Boaventura Souza. As tensões da modernidade. Porto Alegre, 2002. Disponible en: <http://www.dhnet.org.br>. Acceso en: septiembre 2009. 
. Sucedió lo impensable. Revista Nueva América, n. 119, 2008. Disponible en: $<\mathrm{http} / /$ www.novamerica.org.br/revista_digital/L0119/rev_reflexao.asp >. Acceso en: enero 2010.

TAYloR, Sandra; Rizvi, Fazal; Lingard, Bob; Henry, Myriam. Educational policy and the politics of change. London \& New York: Routledge, 1997.

Tello, César; Gorostiaga, Jorge M. El enfoque de la cartografía social para el análisis de debates sobre políticas educativas. Práxis Educativa, v. 4, n. 2, Ponta Grossa, p. 159$-168,2009$.

Tenti fanfani, Emilio. La escuela y la cuestión social. Buenos Aires: Siglo Veintuno Editores, 2007.

TikLY, Leon. Globalisation and education in the postcolonial world. Comparative Education, v. 37, n. 2, p. 151-171, 2001.

Torres, Carlos A. Democracia, educación y multiculturalismo. México: Siglo XXI, 2001. Whitty, Geoff; Power, Sally; Halpin, David. Devolution and choice in education: the school, the state and the market. Buckingham-Philadelphia: Open University Press, 1998.

\section{Anexo}

Viil Conferencia Iberoamericana De Educación. Declaración de Sintra. Sintra, Portugal, 1998. Disponible en: <www.oei.es/viiicie.htm>. Acceso en: enero 2008.

Arrien, Juan. La educación y la reforma de la educación en América Central. In: Navarro, Juan C.; TaYlor, Katherine; Bernasconi, Andrés; TYler, Lewis (Org.), Perspectivas sobre la reforma educativa: América Central en el contexto de políticas de educación en las Américas. USAID/BID/Instituto de Harvard para el Desarrollo Internacional, 2000.

Banco Interamericano de Desarrollo (BID). Hacia la expansión del capital de conocimiento de América Latina y el Caribe: una estrategia del BID para la educación y la capacitación. Borrador, 2005. Disponible en: <www.iadb.org>. Acceso en: junio 2006. Banco mundial. La educación en América Latina y el Caribe. Washington, DC: Banco Mundial, 1999.

Bello, María E. Educación y globalización: los discursos educativos en Iberoamérica. Barcelona: Anthropos, 2003.

Bonal, Xavier. Globalización y política educativa: un análisis crítico de la agenda del Banco Mundial para América Latina. Revista Mexicana de Sociología, v. 64, n. 3, p. 3-35, 2002.

Brunner, José J. Globalización y el futuro de la educación: tendencias, desafíos, estrategias. Seminario sobre prospectivas de la Educación en la Región de América Latina y el Caribe. UNESCO-OREALC, Santiago de Chile, 2000. 
Castro, Claudio de Moura; Carnoy, Martin. Como anda a reforma da educação na América Latina? Rio de Janeiro: Fundação Getulio Vargas, 1997.

CAsassus, Juan. A reforma educacional na América Latina no contexto de globalização. Cadernos de Pesquisa, n. 114, p. 7-28, 2001.

CEPAL. Globalización y desarrollo, Sintesis. Santiago: CEPAL, Secretaría Ejecutiva, 2002.

CEPAL/OEI/Corporación Escenarios(CE). Documento síntesis para la Segunda Conferencia de ex Presidentes de América Latina. In: Educación y globalización: los desafíos para América Latina. 2004. Disponible en: <www.oei.es>. Acceso en: junio 2006. Concha Albornoz, Carlos. Gestión de las reformas educacionales en América Latina en los 90. Primeras aproximaciones a un proceso complejo. Revista Electrónica Iberoamericana sobre Calidad, Eficacia y Cambio en Educación, vol. 3, n. 2, p. 131-53, 2005. CTERA et al. Las reformas educativas en los países del Cono Sur. Algunos resultados y conclusiones de una investigación intersindical. Buenos Aires: LPP, 2005.

Dieterich, Heinz. Globalización, educación y democracia en América Latina. In: Chomsky, Noam; Dieterich, Heinz. La sociedad global. México: Edit. Joaquín Mortiz, 1996.

Donoso Torres, Roberto. Mito y educación: el impacto de la globalización en la educación en América Latina. Buenos Aires: Espacio Editorial, 1999.

FELDFEBER, Myriam. Gestión de la educación, políticas educacionales y globalización, Educação: Teoria e Prática, v. 15, n. 27, p. 13-35, 2006.

Gadotti, Moacir. Ciudadanía planetaria: Puntos para la reflexión. En: Gadotti, M. et al. Perspectivas actuales de la educación. Buenos Aires: Siglo XXI Editores, 2003.

GajARDO, Marcela. Reformas educativas en América Latina. Balance de una década. Cuaderno de trabajo n. 15. Santiago de Chile: Programa de Promoción de la Reforma Educativa en América Latina y el Caribe, 1999. Disponible en:

$<$ http://www.preal.org/Archivos/Bajar.asp?Carpeta=Preal\%20Publicaciones $\backslash$ PREAL \%20Documentos\&Archivo=gajardo15espa\%F1ol.pdf >. Acceso en: junio 2008.

Gallegos, Miguel. La educación en Latinoamérica y el Caribe: puntos críticos y utopías. Revista Latinoamericana de Estudios Educativos, v. 35, n. 1-2, p. 7-34, 2005.

LAMPERT, Ernesto. Educação e Mercosul: desafios e perspectivas. Rev. Fac. Educ. v. 24, n. 2, São Paulo, 1998.

MAUÉs, Olgaises C. Reformas internacionais da educação e formação de professores. Cadernos de Pesquisa, n. 118, p. 89-117, 2003.

Martínez Boom, Alberto. La educación en América Latina: de políticas expansivas a estrategias competitivas. Revista Colombiana de Educación 44, p. 13-39, 2003.

Martínez usarralde, María J. La educación en América Latina: entre la calidad y la equidad. Barcelona: Octaedro, 2005. 
Mejía, Marco R. Cambio curricular y despedagogización en la globalización. Docencia n. 28, p. 40-53, 2006.

Melo, Rosa M. Educación y multilateralismo en América Latina: el tema educativo en la agenda de la Cumbre de las Américas. Revista de Pedagogía, Caracas, v. 29, n. 84, p. 89-122, 2008.

Navarro, Juan C.; Taylor, Katherine; Bernasconi, Andrés; Tyler, Lewis. Prefacio. In: Perspectivas sobre la reforma educativa: América Central en el contexto de políticas de educación en las Américas. USAID/BID/Instituto de Harvard para el Desarrollo Internacional, 2000.

Noriega, Margarita. En la globalización, dos generaciones de reformas educativas en AL: modelos, significados y sujetos. Revista Latinoamericana de Estudios Educativos, v. 37, p. 249-65, 2007.

OEA (Organización de Estados Americanos). Education in the Americas: quality and equity in the globalization process. Washington: Unit for Social Development and Education of the General Secretariat of the Organization of American States, 1997.

OliveirA, Dalila Andrade. Regulação das políticas educacionais na América Latina e suas consequências para os trabalhadores docentes. Educ. Soc., v. 26, n. 92, p. 753-775, 2005.

Ortega Goodspeed, Tamara; Puryear, John. "How competitive are Latin America's schools?”. Focal Point, v. 6 (8), 2007. Disponible en: <http://www.focal.ca/publications/ focalpoint/fp1007/?article=article2\&lang=s $>$. Acceso en: septiembre 2008.

Ottone, Ernesto. La apuesta educativa en América Latina. In: Rojas Aravena, Francisco (Org.) Globalización, América Latina y la diplomacia de cumbres. Santiago: FLACSO, 1998.

; Hopenhayn, Martin. Desafíos educativos ante la sociedad del conocimiento. Revista Pensamiento Educativo, v. 40, n. 1, p. 13-29, 2007.

Preal (Programa de Promoción de la Reforma Educativa en América Latina y el Caribe). Quedándonos atrás: un informe del progreso educativo en América Latina. 2001. Disponible en: <http://www.preal.org >. Acceso en: mayo 2006.

. El futuro está en juego. 1998. Disponible en: <http://www.preal.org>. Acceso en: mayo 2006.

Rambla, Xavier. Globalization, educational targeting, and stable inequalities: a comparative analysis of Argentina, Brazil, and Chile. International Review of Education, n. 52, p. 353-370, 2006.

Rigal, Luis. El sentido de educar. Crítica a los procesos de transformación educativa en Argentina dentro del marco latinoamericano. Buenos Aires: Miño y Davila, 2004.

Rivero, José. Educación y exclusión en América Latina: reformas en tiempo de globalización. Madrid: Miño y Dávila, 1999. 
Schwartzman, Simon. El futuro de la educación en America Latina y el Caribe. Ed-01/ promedlac vii/ref.2. Documento de Trabajo, 2001.

Stromeuist, Nelly. While gender sleeps. In: Ball, Stephen; Fischman, Gustavo; GvirTz, Silvina (Org.). Crisis and Hope: The Educational Hopscotch of Latin America. London: Routledge-Falmer, 2003.

Tedesco, Juan C. Educar en la sociedad del conocimiento. Buenos Aires: FCE, 2000.

Tiramonti, Guillermina. Los imperativos de las políticas educativas de los '90. Revista da Faculdade de Educação, São Paulo, v. 23, n. 1-2, 1997.

Torres, Carlos A. Educación, globalización y justicia. Conferencia pronunciada en el Centro SocioCultural Caixa Galicia A Coruña, España, 2007.

. The State, privatisation and educational policy: a critique of neo-liberalism in Latin America and some ethical and political implications. Comparative Education, v. 38, n. 4, p. 365-85, 2002.

Unesco/Orealc. Situación educativa de América Latina y el Caribe. 1980-2000. Santiago de Chile: Oficina Regional de Educación para América Latina y el Caribe, UNESCO, 2001.

. La UNESCO y la educación en América Latina y el Caribe 1987-1997. Santiago de Chile: Oficina Regional de Educación para América Latina y el Caribe, UNESCO, 1998.

\section{SOBRE LOS AUTORES}

Jorge M. Gorostiaga es doctor en educación comparada (University of Pittsburgh). Profesor asociado (Escuela de Humanidades, Universidad Nacional de San Martín) e investigador del Consejo Nacional de Investigaciones Científicas y Técnicas.

E-mail: jorgegoros@gmail.com

CÉSAR G. TELLO es magíster en políticas y administración de la educación (Universidad Nacional de Tres de Febrer) y diplomado superior en gestión educativa por FLACSO-Argentina. Docente de las Universidades Nacionales de La Plata, Tres de Febrero y San Martín.

E-mail: cesargeronimotello@yahoo.com.ar

Recebido em fevereiro de 2011 Aprovado em abril de 2011 
Globalização e reformas educacionais na América Latina: uma análise inter-textual

Este artigo trata sobre as relações entre os processos de globalização e as reformas educacionais implementadas na América Latina nas últimas duas décadas. Reportamos uma pesquisa que procurou caracterizar e comparar as principais perspectivas sobre este tema através da análise de textos produzidos por acadêmicos e por organizações governamentais e não governamentais. Empregando a metodologia da cartografia social, combinam-se ferramentas da análise textual com uma representação visual do discurso como campo intertextual. No mapeamento, identificamos sete perspectivas principais: Economicista, Inserção Imperativa, Integracionista, Crítica Normativa, Crítica Analítica e Mundialização Alternativa. 
Palavras-chave: globalização; reformas educacionais; América Latina; cartografia social

\section{Globalization and educational reform in Latin America: an inter-textual analysis}

This article addresses the relationships between globalization processes and the education reforms implemented in Latin America during the last two decades. We report a study that aimed at characterizing and comparing the main perspectives on this issue through the analysis of texts produced by academics and by governmental and non-governmental organizations. Employing the methodology of social cartography, we combine textual analysis tools with a visual representation of the discourse as an intertextual field. In our mapping we identify seven main perspectives: Economicist, Imperative Insertion, Integrationist, Normative Critique, Analytic Critique, and Alternative Globalization.

Keywords: globalization; educational reforms; Latin America; social cartography

\section{Globalización y reforma educativa en América Latina: un análisis inter-textual}

Este artículo aborda la relación entre los procesos de globalización y las reformas educativas que han tenido lugar en América Latina durante las últimas dos décadas. Damos cuenta de una investigación que buscó caracterizar y comparar las principales perspectivas sobre esta temática a través del análisis de textos producidos por académicos y por organizaciones gubernamentales y no gubernamentales. Siguiendo la metodología de la cartografía social, se combinan herramientas del análisis textual con una representación visual del discurso como campo intertextual. En el mapeo que presentamos, hemos identificado siete perspectivas principales: Economicista, Inserción Imperativa, Integracionista, Humanista, Crítica Normativa, Crítica Analítica y Mundialización Alternativa.

Palabras clave: globalización; reformas educativas; América Latina; cartografía social 